\title{
Imprints of cosmic strings on the cosmological gravitational wave background
}

\author{
K Kleidis, ${ }^{1,2}$ D B Papadopoulos, ${ }^{1}$ E Verdaguer, ${ }^{3}$ and L Vlahos ${ }^{1}$ \\ ${ }^{1}$ Department of Physics, Aristotle University of Thessaloniki, 54124 Thessaloniki, Greece \\ ${ }^{2}$ Department of Civil Engineering, Technological Education Institute of Serres, 62124 Serres, Greece \\ ${ }^{3}$ Departament de Fisica Fonamental and Institut de Ciences del Cosmos, Universitat de Barcelona, Avinguda Diagonal 647, \\ E-08028 Barcelona, Spain
}

(Received 6 March 2008; published 16 July 2008)

\begin{abstract}
The equation which governs the temporal evolution of a gravitational wave $(\mathrm{GW})$ in curved space-time can be treated as the Schrödinger equation for a particle moving in the presence of an effective potential. When GWs propagate in an expanding universe with constant effective potential, there is a critical value $\left(k_{c}\right)$ of the comoving wave number which discriminates the metric perturbations into oscillating $\left(k>k_{c}\right)$ and nonoscillating $\left(k<k_{c}\right)$ modes. As a consequence, if the nonoscillatory modes are outside the horizon they do not freeze out. The effective potential is reduced to a nonvanishing constant in a cosmological model which is driven by a two-component fluid, consisting of radiation (dominant) and cosmic strings (subdominant). It is known that the cosmological evolution gradually results in the scaling of a cosmicstring network and, therefore, after some time $(\Delta \tau)$ the Universe becomes radiation dominated. The evolution of the nonoscillatory GW modes during $\Delta \tau$ (while they were outside the horizon), results in the distortion of the GW power spectrum from what it is anticipated in a pure radiation model, at present-time frequencies in the range $10^{-16} \mathrm{~Hz}<f \leqq 10^{5} \mathrm{~Hz}$.
\end{abstract}

DOI: 10.1103/PhysRevD.78.024027

PACS numbers: 04.30.-w, 11.25.-w, 98.80.Cq

\section{INTRODUCTION}

The so-called cosmological gravitational waves (CGW) represent small-scale perturbations to the Universe metric tensor [1]. Since gravity is the weakest of the four known forces, these metric corrections decouple from the rest of the Universe at very early times, presumably at the Planck epoch [2]. Their subsequent propagation is governed by the space-time curvature, encapsulating in the field equations the inherent coupling between relic GWs and the Universe matter content; the latter being responsible for the background gravitational field [3].

In this context, we consider the coupling between CGWs and cosmic strings. They are one-dimensional objects that can be formed as linear defects at a symmetry-breaking phase transition $[4,5]$. If they exist, they may help us to explain some of the large-scale structures seen in the Universe today, such as gravitational lenses [6]. They may also serve as seeds for density perturbations [7,8], as well as potential sources of relic gravitational radiation [9].

In the present article we explore another possibility: A fluid of cosmic strings could be responsible for the constancy of the effective potential in the equation which drives the temporal evolution of a CGW in an expanding universe. As we find out, a constant effective potential leads to a critical comoving wave number $\left(k_{c}\right)$, which discriminates the metric fluctuations into oscillating modes $\left(k>k_{c}\right)$ and nonoscillatory $\left(k<k_{c}\right)$ ones. As long as the latter lie outside the horizon, they do not freeze out, resulting in the departure of the inflationary-GW power spectrum from scale invariance. This would be the case, if there is a short period after inflation where the cosmologi- cal fluid is made out of radiation and a subdominant component of cosmic strings. As regards the space-time geometry itself, the spatially flat Friedmann-RobertsonWalker (FRW) model appears to interpret adequately both the observational data related to the known thermal history of the Universe and the theoretical approach to cosmic-string configurations [4]. Consequently, we will assume our cosmological background to be a spatially flat FRW model.

This paper is organized as follows: In Sec. II we summarize the theory of CGWs in curved space-time. In Sec. III we demonstrate that, in a radiation model contaminated by a fraction of cosmic strings, the effective potential in the equation which governs the temporal evolution of a CGW in curved space-time is constant. In Sec. IV we explore the characteristics of a potential contribution of cosmic strings to the evolution of the Universe and in Sec. V we study the propagation of the nonoscillatory GW modes during this stage. We find that, if the Universe evolution includes a radiation-plus-strings stage, then, although it could last only for a short period of time, its presence would lead to a distortion of the stochastic GW background from what it is anticipated in a pure radiation model, at present-time frequencies in the range $10^{-16} \mathrm{~Hz}<f \leqq 10^{5} \mathrm{~Hz}$.

\section{GRAVITATIONAL WAVES IN CURVED SPACE-TIME}

The far-field propagation of a weak CGW $\left(\left|h_{\mu \nu}\right| \ll 1\right)$ in a curved, nonvacuum space-time is determined by the differential equations [10] 


$$
h_{\mu \nu ; \alpha}^{; \alpha}-2 \mathcal{R}_{\alpha \mu \nu \beta} h^{\alpha \beta}=0
$$

under the gauge choice

$$
\left(h^{\alpha \beta}-\frac{1}{2} g^{\alpha \beta} h_{\mu}^{\mu}\right)_{; \beta}=0
$$

which brings the linearized Einstein equations into the form (1). In Eqs. (1) and (2), Greek indices refer to the four-dimensional space-time, $\mathcal{R}_{\alpha \mu \nu \beta}$ is the Riemann curvature tensor of the background metric, $h_{\mu}^{\mu}$ is the trace of $h_{\mu \nu}$ and the semicolon denotes the covariant derivative.

In the system of units where $c=1$, a linearly-polarized, plane GW propagating in a spatially flat FRW cosmological model, is defined as [9]

$$
d s^{2}=R^{2}(\tau)\left[d \tau^{2}-\left(\delta_{i j}+h_{i j}\right) d x^{i} d x^{j}\right]
$$

where $\tau$ is the conformal-time coordinate, Latin indices refer to the three-dimensional spatial section, and $\delta_{i j}$ is the Kronecker symbol. The dimensionless scale factor $R(\tau)$ is a solution to the Friedmann equation

$$
\left(\frac{R^{\prime}}{R^{2}}\right)^{2}=\frac{8 \pi G}{3} \rho(\tau)
$$

(where, the prime denotes differentiation with respect to $\tau$ and $G$ is Newton's constant), with matter content in the form of a perfect fluid, $T_{\mu \nu}=\operatorname{diag}(\rho,-p,-p,-p)$, which obeys the conservation law

$$
\rho^{\prime}+3 \frac{R^{\prime}}{R}(\rho+p)=0
$$

and the equation of state

$$
p=\left(\frac{m}{3}-1\right) \rho
$$

where $\rho(\tau)$ and $p(\tau)$ represent the mass density and the pressure, respectively.

The linear equation of state (6) covers most of the matter components considered to drive the evolution of the Universe [11-13], such as a quantum vacuum $(m=0)$, a network of domain walls $(m=1)$, a gas of cosmic strings $(m=2)$, dust $(m=3)$, radiation $(m=4)$, and Zel'dovich ultrastiff matter $(m=6)$. For each component, the continuity Eq. (5) yields

$$
\rho=\frac{M_{m}}{R^{m}}
$$

where $M_{m}$ is an integration constant, associated to the initial mass density of the $m$ th component. Provided that the various components do not interact with each other, a mixture of them obeys [11]

$$
\rho=\sum_{m} \frac{M_{m}}{R^{m}}
$$

where, now, Eq. (5) holds for each matter constituent separately.
In the case of a one component fluid, the Friedmann Eq. (4) reads

$$
R^{(m / 2)-2} R^{\prime}=\left(\frac{8 \pi G}{3} M_{m}\right)^{1 / 2}
$$

and, for every type of matter content other than cosmic strings $(m \neq 2)$, it results in

$$
R(\eta)=\left(\frac{\eta}{\eta_{m}}\right)^{2 /(m-2)}
$$

where, the time parameter $\eta$ is linearly related to the corresponding conformal one, by $\eta=\frac{m-2}{2} \tau$ and we have set $\eta_{m}=\left(\frac{8 \pi G}{3} M_{m}\right)^{-1 / 2}$. Notice that, for $m=0$ (de Sitter inflation) and $0<\tau<\infty$, we obtain $-\infty<\eta<0$.

The general solution to Eq. (1) in the curved space-time (3) is a linear superposition of plane-wave modes

$$
h_{i j}(\tau, \vec{x})=\frac{h_{k}(\tau)}{R(\tau)} \varepsilon_{i j} e^{i k_{j} x^{j}}
$$

where $h_{k}(\tau)$ is the time-dependent part of the mode denoted by $k$ and $\varepsilon_{i j}$ is the polarization tensor, depending only on the direction of the comoving wave vector $k_{j}$. Accordingly, for a fixed wave number $k^{2}=\sum k_{j}^{2}$, the time-dependent part of the corresponding GW mode satisfies the second-order differential equation $[14,15]$

$$
h_{k}^{\prime \prime}(\tau)+\left(k^{2}-\frac{R^{\prime \prime}}{R}\right) h_{k}(\tau)=0 .
$$

Equation (12) can be treated as the Schrödinger equation for a particle moving in the presence of the effective potential

$$
V_{\text {eff }}=\frac{R^{\prime \prime}}{R}
$$

and, in a cosmological model of the form (13), is written in the form

$$
h^{\prime \prime}(\eta)+\left\{k_{m}^{2}-2\left[\frac{4-m}{(m-2)^{2}}\right] \frac{1}{\eta^{2}}\right\} h(\eta)=0
$$

yielding

$$
h_{m}\left(k_{m} \eta\right)=\sqrt{\eta}\left[c_{1} H_{|\nu|}^{(1)}\left(k_{m} \eta\right)+c_{2} H_{|\nu|}^{(2)}\left(k_{m} \eta\right)\right]
$$

where, now, a prime denotes the derivative with respect to $\eta, c_{1}$, and $c_{2}$ are arbitrary constants to be determined by the initial conditions and $k_{m}=\frac{2}{m-2} k$, so that $k_{m} \eta=k \tau$. Finally, $H_{|\nu|}^{(1)}$ and $H_{|\nu|}^{(2)}$ are the Hankel functions of the first and the second kind, of order [16]

$$
|\nu|=\frac{1}{2}\left|\frac{m-6}{m-2}\right| \text {. }
$$

Therefore, different types of matter content (reflecting different periods in the evolution of the Universe) admit different Hankel functions (see, also, [17]). 


\section{CONSTANCY OF THE EFFECTIVE POTENTIAL}

\section{A. Implications on CGW's propagation}

A case of particular interest, involved in the time evolution of a primordial $\mathrm{GW}$, is when the effective potential (13) is constant for every $\tau$, namely

$$
\frac{R^{\prime \prime}}{R}=\frac{8 \pi G}{3} M
$$

where $M$ is a non-negative constant of dimensions $L^{-4}$. In this case, Eq. (12) is written in the form

$$
h_{k}^{\prime \prime}(\tau)+\omega^{2} h_{k}(\tau)=0
$$

where

$$
\omega^{2}=k^{2}-\frac{8 \pi G}{3} M
$$

is the (constant) frequency of the wave. According to Eq. (19), a critical value of the comoving wave number arises, through the condition

$$
\omega^{2} \gtrless 0 \Leftrightarrow k \gtrless k_{c}=\sqrt{\frac{8 \pi G}{3} M .}
$$

This critical value discriminates the primordial GWs in modes with $k>k_{c}$, which oscillate for every $\tau$,

$$
h_{k>k_{c}}(\tau) \sim e^{l \sqrt{k^{2}-k_{c}^{2}} \tau}
$$

and modes with $k<k_{c}$, which grow exponentially for every $\tau$,

$$
h_{k<k_{c}}(\tau) \sim e^{\sqrt{k_{c}^{2}-k^{2}} \tau}
$$

(the exponentially decaying solutions are neglected).

\section{B. Cosmological models of constant effective potential}

Now, the question arises on whether there exists a spatially flat FRW cosmological model in which the effective potential is constant. To answer this question, we set $\mathcal{H}=$ $\frac{R^{\prime}}{R}$. Accordingly, $V_{\text {eff }}$ is written in the form

$$
\frac{R^{\prime \prime}}{R}=\mathcal{H}^{\prime}+\mathcal{H}^{2} .
$$

Upon consideration of Eqs. (4) and (23), Eq. (17) results in the ordinary differential equation

$$
\rho^{\prime}+4 \rho \frac{R^{\prime}}{R}=2 M \frac{R^{\prime}}{R^{3}}
$$

which admits the solution

$$
\rho(\tau)=\frac{C}{R^{4}}+\frac{M}{R^{2}}
$$

where $C$ is an arbitrary integration constant of dimensions
$L^{-4}$. In comparison to Eqs. (7) and (8), we distinguish the following cases:

(i) $\quad M=0$ and $C=0$ : This case corresponds to vacuum and flat space-time and it will not be considered further.

(ii) $\quad M=0$ and $C \neq 0$ : This choice results in a radiation-dominated universe

$$
\rho(\tau)=\frac{C}{R^{4}}, \quad V_{\text {eff }}=0
$$

where the critical wave number vanishes $\left(k_{c}=0\right)$.

(iii) $\quad M \neq 0$ and $C=0$ : Hence,

$$
\rho(\tau)=\frac{M}{R^{2}}, \quad V_{\text {eff }}=\frac{8 \pi G}{3} M
$$

which corresponds to a string-dominated universe [18]. It is worth noting that the constant $M$ appearing in the effective potential (17) is, in fact, the initial mass density of the strings, $M_{2}$ [cf. Eq. (7)]. A string-dominated universe does not seem likely $[19,20]$ and, therefore, this case is of no particular interest.

(iv) Finally, if both $C$ and $M$ differ from zero, then, the function $\rho(\tau)$ consists of two parts: One evolving as $R^{-4}$ and the other as $R^{-2}$. By analogy to Eq. (8), this type of matter content can be met in a cosmological model filled with relativistic particles (radiation) and a fluid of cosmic strings, without interacting with each other, as it should be the case shortly after the dynamic friction between them [4] became unimportant [5]. Therefore, in this case,

$$
\rho=\frac{M_{4}}{R^{4}}+\frac{M_{2}}{R^{2}}, \quad V_{\mathrm{eff}}=\frac{8 \pi G}{3} M_{2} .
$$

Once again, the constant $M$, appearing in the effective potential, is associated to the initial amount of strings in the mixture. It appears that, whenever the effective potential acquires a nonzero constant value, this value always involves the initial density of a cosmic-string gas.

We conclude that, in the presence of cosmic strings the effective potential is reduced to a nonvanishing constant and, therefore, oscillation of the metric perturbations is possible only if their comoving wave number is larger than a critical value, depending on the mass density of the linear defects

$$
k>k_{c}=\sqrt{\frac{8 \pi G}{3} M_{2}} .
$$

In other words, a cosmic-string network discriminates the primordial GWs predicted by inflation into oscillating and nonoscillating modes, something that should be reflected in the power spectrum of the stochastic GW background. We shall attempt to illustrate how, in a realistic setting. 


\section{A UNIVERSE WITH COSMIC STRINGS}

The presence of cosmic strings in a unified gauge theory is purely a question of topology. The simplest $\mathrm{SO}(10)$ model, for example, predicts strings [21]. Many superstring-inspired models also result in the formation of linear topological defects [22,23]. Cosmic strings are formed at a symmetry-breaking phase transition, within the radiation-dominated epoch

$$
R(\tau)=\frac{\tau}{\tau_{c r}}
$$

where $\tau_{c r}$ is the time at which the Universe acquires the critical temperature below which the strings are formed and we have normalized $R\left(\tau_{c r}\right)$ to unity.

In particular, after inflation (and reheating) the Universe enters in an early-radiation epoch [24], during which the background temperature drops monotonically $\left(T \sim R^{-1}\right)$. For $\tau \geq \tau_{c r}$, this cooling process results in the breaking of a fundamental U(1) local gauge symmetry, leading to the formation of linear defects (for a detailed analysis see [4] and/or [5]).

By the time the cosmic strings are formed, they are moving in a very dense environment and, hence, their motion is heavily damped due to string-particle scattering [25-28]. This friction becomes subdominant to expansion damping at [25]

$$
\tau_{*}=\frac{1}{\sqrt{G \mu}} \tau_{c r}
$$

where, $\mu$ is the mass per unit length of the linear defect. For $\tau \geq \tau_{*}$, the motion of long cosmic strings can be considered essentially independent of anything else in the Universe and soon they acquire relativistic velocities. Therefore, we may consider that, after $\tau_{*}$ the evolution of the Universe is driven by a two-component fluid, consisting of relativistic particles (dominant) and cosmic strings (subdominant). Consequently, Eq. (28) holds and $\tau_{*}$ marks the beginning of a radiation-plus-strings stage. During this stage, the Friedmann Eq. (4) yields

$$
R(\tau)=\sqrt{\frac{M_{4}}{M_{2}}} \sinh \sqrt{\frac{8 \pi G}{3} M_{2}} \tau .
$$

Nevertheless, the scale factor (32) can drive the Universe expansion only for a short period of time after $\tau_{*}$, since cosmic strings should (at any time) be a small proportion of the Universe energy content. This means that the equation of state considered in (28) should have validity only for a limited time period, otherwise cosmic strings would eventually dominate the overall energy density [18].

In fact, a radiation-plus-strings stage (if ever existed) does not last very long. Numerical simulations [29-32] suggest that, after the friction becomes unimportant, the production of loops smaller than the Hubble radius gradually results in the scaling of the long-string network.
Accordingly, the linear defects form a self-similar configuration, the density of which, eventually, behaves as $R^{-4}$ [4]. In this way, apart from small statistical fluctuations, at some time $\tau_{s c}>\tau_{*}$ the Universe reenters in the (late) radiation era

$$
R(\tau)=R_{s c} \frac{\tau}{\tau_{s c}}
$$

before it can become string dominated. The duration $\left(\Delta \tau=\tau_{s c}-\tau_{*}\right)$ of the radiation-plus-strings stage is quite uncertain, mostly due to the fact that numerical simulations can be run for relatively limited times. For example, the longest run of [32] suggests that $\tau_{s c} \simeq 4.24 \tau_{*}$ (corresponding to a factor of 18 in terms of the physical time), while [31] raises this value to $\tau_{s c} \simeq 6.48 \tau_{*}\left(t_{s c} \simeq 42 t_{*}\right)$.

In what follows, we explore the evolution of GW modes with $k<k_{c}$ through the radiation-plus-strings stage.

\section{CGWS IN THE PRESENCE OF COSMIC STRINGS}

\section{A. Evolution of modes outside the horizon}

CGWs are produced by quantum fluctuations during inflation (e.g., see [33]). Some of them escape from the visible Universe, once their reduced physical wavelength $\left[\lambda_{\mathrm{ph}}=\frac{\lambda}{2 \pi} R(\tau)\right]$ becomes larger than the (constant) inflationary horizon $\left[\ell_{H}=H_{d S}^{-1}, H_{d S}\right.$ being the Hubble parameter of the de Sitter space]. Eventually, every CGW with $k \leq k_{\max }=H_{d S} R_{d S}$ is exiled from the Hubble sphere and freezes out, acquiring the constant amplitude [34], [35]

$$
\alpha_{k}^{2}=\left[\frac{h_{k}(\tau)}{R(\tau)}\right]^{2}=\frac{16}{\pi}\left(\frac{H_{d S}}{m_{P l}}\right)^{2} k^{-3}
$$

where $m_{\mathrm{Pl}}=G^{-1 / 2}$ is the Planck mass. After inflation, i.e., within the subsequent radiation epoch, analytic solutions for $\alpha_{k}(\tau)$ can be expressed in terms of the Bessel function $J_{1 / 2}(k \tau)$

$$
\alpha_{k}(\tau)=2 c_{1} \alpha_{k} \frac{\sqrt{\tau}}{R(\tau)} J_{1 / 2}(k \tau) \sim \alpha_{k} \frac{\sin k \tau}{k \tau}
$$

[cf. Eq. (15) for $c_{1}=c_{2}$ ]. Accordingly, when $k \tau \ll 1$, the perturbation's amplitude evolves slowly and is approximately constant. Once $k \tau \approx 1$, the amplitude decays away rapidly before entering in an oscillatory phase with slowly decreasing amplitude, when $k \tau \gg 1$. Physically, this corresponds to a mode that is (almost) frozen beyond the horizon, until its physical wavelength becomes comparable to the Hubble radius, at which point it enters in the visible Universe (e.g., see [36]).

In other words, as the Universe expands, a fraction of the modes that lie beyond the horizon reenters inside the Hubble sphere. At the time of reentry their amplitude is given by Eq. (34), while, afterwards, they begin oscillating. The $k$ dependence of their amplitude implies a scaleinvariant power spectrum [37]. 
However, if the cosmological evolution includes a radiation-plus-strings stage, then, during this stage, the effective potential is a nonvanishing constant. In other words, $k_{c} \neq 0$ and the equation which governs the temporal evolution of the GW modes with $k<k_{c}$ does not admit the solution (35), but Eq. (22). As a consequence, even if they lie outside the horizon, these modes do not freeze out.

At the beginning of the radiation-plus-strings stage, the GW modes that fit inside the visible Universe obey the condition

$$
\lambda_{\mathrm{ph}}\left(\tau_{*}\right) \leq \ell_{H}\left(\tau_{*}\right) \Rightarrow k \geq H\left(\tau_{*}\right) R\left(\tau_{*}\right)=\frac{1}{\tau_{*}}
$$

while, modes of $k<k_{*}=\tau_{*}^{-1}$ lie outside the horizon. In order to examine whether $k_{c} \gtrless k_{*}$ we need to determine the initial mass density of the linear defects, since, by definition,

$$
M_{2}=\rho_{\mathrm{str}}\left(\tau_{*}\right) R^{2}\left(\tau_{*}\right) .
$$

Let us consider a network of cosmic strings characterized by a correlation length $\xi(\tau)$. This may be defined as the length such that the mass within a typical volume $\xi^{3}$, is $\mu \xi$ [4]. In this case, at $\tau=\tau_{*}$, the cosmic strings contribute to the Universe matter content a mean density

$$
\rho_{\text {str }}\left(\tau_{*}\right)=\frac{\mu}{\xi^{2}\left(\tau_{*}\right)}=\gamma_{*}^{2} \frac{\mu}{\ell_{H}^{2}\left(\tau_{*}\right)}
$$

where $\gamma_{*}$ is a numerical constant of the order of unity, representing the number of correlation lengths inside the horizon at $\tau_{*}$. Accordingly,

$$
M_{2}=\mu\left(\frac{\gamma_{*}}{\tau_{*}}\right)^{2}
$$

and hence

$$
k_{c}=\sqrt{\frac{8 \pi}{3}} \sqrt{G \mu} \frac{\gamma_{*}}{\tau_{*}} .
$$

For grand unified theory (GUT)-scale strings we have $(G \mu) \sim 10^{-6}$ and $\gamma_{*} \simeq 7$ (e.g., see [4]), so that $k_{c} \simeq 2 \times$ $10^{-2} k_{*} \ll k_{*}$. In other words, at the beginning of the radiation-plus-strings stage, the GW modes of $k<k_{c}$ do not fit inside the horizon.

On the other hand, for $\tau_{*}<\tau \leq \tau_{s c}$, the condition of fitting inside the Hubble sphere is written in the form

$$
\frac{k}{k_{c}} \geq \operatorname{coth} \sqrt{\frac{8 \pi G}{3} M_{2} \tau}
$$

Since the hyperbolic cotangent on the right-hand side is larger than unity for every $\tau$, Eq. (41) suggests that the GW modes of comoving wave numbers $k<k_{c}$ remain outside the horizon during the whole radiation-plus-strings stage.

Nevertheless, by virtue of Eq. (22), for $\tau_{*}<\tau \leq \tau_{s c}$ their amplitude continues to evolve as

$$
\alpha_{k<k_{c}}\left(\tau>\tau_{*}\right)=\frac{4}{\sqrt{\pi}}\left(\frac{H_{d S}}{m_{P l}}\right) \frac{1}{k^{3 / 2}}\left[\frac{R\left(\tau_{*}\right)}{R(\tau)}\right] e^{\sqrt{k_{c}^{2}-k^{2}}\left(\tau-\tau_{*}\right)}
$$

[cf. Eqs. (22) and (34)]. This behavior ends at $\tau_{s c}$, when the scaling of the long-string network is completed and the Universe reenters in the (late) radiation era. For $\tau>\tau_{s c}$ the GW modes of $k<k_{c}$ are no longer influenced by the radiation-plus-strings stage and therefore, just like the rest of the metric perturbations outside the horizon, (re) freeze out. As a consequence, their amplitude acquires the constant value

$$
\alpha_{k<k_{c}}=\frac{4}{\sqrt{\pi}}\left(\frac{H_{d S}}{m_{P l}}\right) \frac{1}{k^{3 / 2}}\left[\frac{R\left(\tau_{*}\right)}{R\left(\tau_{s c}\right)}\right] e^{\sqrt{k_{c}^{2}-k^{2}} \Delta \tau} .
$$

\section{B. The distorted power spectrum}

Within the late-radiation era these modes remain frozen until the time $\tau_{c}$. At that time the mode $k_{c}$ enters inside the visible Universe, since its physical wavelength $\left(\lambda_{c_{\mathrm{ph}}} \sim \tau_{c}\right)$ becomes smaller than the corresponding Hubble radius $\left(\ell_{H} \sim \tau_{c}^{2}\right)$. In accordance, for $\tau>\tau_{c}$, GW modes of $k<$ $k_{c}$ also enter inside the Hubble sphere. After entering inside the horizon, the GW modes under consideration begin oscillating, thus producing a part of the power spectrum we observe today (or at some time in the future). However, since they have experienced the influence of the radiationplus-strings (rps) stage, their amplitude is no longer given by Eq. (34), but by Eq. (43), thus resulting in the distortion of the GW power spectrum $\left(P_{k}^{2} \sim k^{3} \alpha_{k}^{2}\right)$, from what it is anticipated by pure-radiation (rad), at comoving wave numbers $k<k_{c}$. Namely,

$$
P_{k<k_{c}}^{\mathrm{rps}}=P_{k<k_{c}}^{\mathrm{rad}} \frac{R\left(\tau_{*}\right)}{R\left(\tau_{s c}\right)} e^{\sqrt{k_{c}^{2}-k^{2}} \Delta \tau}
$$

which, upon consideration of Eq. (32), is written in the form

$$
\frac{P_{k<k_{c}}^{\mathrm{rps}}}{P_{k<k_{c}}^{\mathrm{rad}}}=\frac{2 e^{\left(1+\sqrt{1-x^{2}}\right) k_{c} \Delta \tau}}{\left[\operatorname{coth}\left(k_{c} \tau_{*}\right)+1\right] e^{2 k_{c} \Delta \tau}-\left[\operatorname{coth}\left(k_{c} \tau_{*}\right)-1\right]}
$$

where, we have set $0<\frac{k}{k_{c}}=x=\frac{f}{f_{c}}<1$ and $f$ is the frequency attributed to the GW mode denoted by $k$. According to Eq. (40), $\operatorname{coth}\left(k_{c} \tau_{*}\right) \simeq 5$ and Eq. (45) result in

$$
\frac{P_{k<k_{c}}^{\mathrm{rps}}}{P_{k<k_{c}}^{\mathrm{rad}}}=\frac{e^{\left(1+\sqrt{1-x^{2}}\right) k_{c} \Delta \tau}}{3 e^{2 k_{c} \Delta \tau}-2} .
$$

Clearly, for $\Delta \tau=0$ (i.e., in the absence of the radiationplus-strings stage) $P_{k<k_{c}}^{\mathrm{rps}}=P_{k<k_{c}}^{\mathrm{rad}}$, while, for $\Delta \tau \neq 0$ the inflationary-GW power spectrum is no longer scale invariant.

The spectral function $\Omega_{\mathrm{gw}}$, appropriate to describe the intensity of a stochastic GW background [38], is related to 
the power spectrum as $\Omega_{\mathrm{gw}} \sim P_{k}^{2}$ (e.g., see [39]). Therefore, Eq. (46) yields

$$
\frac{\Omega_{g w}^{\mathrm{rps}}\left(f<f_{c}\right)}{\Omega_{\mathrm{gw}}^{\mathrm{rad}}\left(f<f_{c}\right)}=\frac{e^{2\left(1+\sqrt{1-x^{2}}\right) k_{c} \Delta \tau}}{\left(3 e^{2 k_{c} \Delta \tau}-2\right)^{2}} .
$$

Notice that, for every $0 \leq x \leq 1$, we have $\Omega_{\mathrm{gw}}^{\mathrm{rps}} \leq \Omega_{\mathrm{gw}}^{\mathrm{rad}}$, with the equality being valid only for $\Delta \tau=0$. In other words, the involvement of a radiation-plus-strings stage in the evolution of the Universe reduces the stochastic GW intensity to lower levels than those expected by pure radiation. To give some numbers, we take into account the numerical results of [31], as well as those of [32]. Accordingly, a reasonable estimate on the duration of radiation-plus-strings stage would be $\tau_{s c}=5.5 \tau_{*}$ and therefore, $k_{c} \Delta \tau \simeq 9 \times 10^{-2}$. In this case, Eq. (47) is written in the form

$$
\frac{\Omega_{g w}^{\mathrm{rps}}\left(f<f_{c}\right)}{\Omega_{\mathrm{gw}}^{\mathrm{rad}}\left(f<f_{c}\right)} \simeq 0.47 \times e^{0.18 \sqrt{1-x^{2}}}
$$

from which it becomes evident that, for $f<f_{c}$, the value of $\Omega_{\mathrm{gw}}$ is no longer $8 \times 10^{-14}$, as it is predicted by pure radiation [2], [9], but rather

$$
\Omega_{\mathrm{gw}}^{\mathrm{rps}} \simeq 0.5 \Omega_{\mathrm{gw}}^{\mathrm{rad}} \simeq 4 \times 10^{-14} \text {. }
$$

Such a distortion reflects a change in the distribution of the GW energy density among the various frequency intervals, probably due to the coupling between metric perturbations and cosmic strings.

The question that arises now is, whether these results are observable by the detectors currently available. To answer this question, we should determine explicitly both $f_{c}$ (the critical frequency) and $t_{c}$ (the physical time at which the GW modes of $f<f_{c}$ begin entering inside the horizon). In what follows, $c \neq 1$.

During the early-radiation epoch, the physical time is defined as

$$
t=\int R(\tau) d \tau \Rightarrow t=\frac{\tau^{2}}{2 \tau_{c r}} .
$$

With the aid of Eqs. (31) and (50), Eq. (40) is written in the form

$$
k_{c}=\sqrt{\frac{2 \pi}{3}}\left(\frac{G \mu}{c^{2}}\right) \frac{\gamma_{*}}{c t_{c r}}
$$

and therefore

$$
f_{c}=\frac{1}{\sqrt{6 \pi}}\left(\frac{G \mu}{c^{2}}\right) \frac{\gamma_{*}}{t_{c r}} .
$$

The first of the GW modes under consideration which enters inside the visible Universe is the one with the shortest comoving wavelength $\left(\lambda_{c}\right)$, i.e., the one with the largest frequency $\left(f_{c}\right)$. In terms of the physical time, this process begins at $t_{c}$, at which $\lambda_{c_{\mathrm{ph}}}\left(t_{c}\right) \leq \ell_{H}\left(t_{c}\right)$.
Within the late-radiation era, the physical time is defined as

$$
t=\int R(\tau) d \tau \Rightarrow t=R_{s c} \frac{\tau^{2}}{2 \tau_{s c}}
$$

and therefore

$$
t_{c} \gtrsim \frac{3 \pi}{11 \gamma_{*}^{2}}\left(\frac{G \mu}{c^{2}}\right)^{-1} t_{*}=\frac{3 \pi}{11 \gamma_{*}^{2}}\left(\frac{G \mu}{c^{2}}\right)^{-2} t_{c r}
$$

where we have used Eq. (31) and the fact that, in an expanding universe,

$$
R_{s c}>R\left(t_{*}\right)=\left(\frac{G \mu}{c^{2}}\right)^{-1 / 2} .
$$

Within the Hubble sphere the GW modes of $\lambda>\lambda_{c}$ correspond to CGWs of frequencies $f<f_{c}$. Extrapolation of this result into the present epoch $\left(t_{\mathrm{pr}} \simeq 13.7 \times 10^{9} y\right)$, suggests that at frequencies

$$
\begin{aligned}
& f^{p r}<f_{c}^{p r}=f_{c}\left(\frac{t_{c}}{t_{\mathrm{rec}}}\right)^{1 / 2}\left(\frac{t_{\mathrm{rec}}}{t_{p r}}\right)^{2 / 3} \Rightarrow \\
& f^{p r}<f_{c}^{p r}=\frac{1}{\sqrt{22}}\left(\frac{t_{c r}}{t_{\mathrm{rec}}}\right)^{1 / 2}\left(\frac{t_{\mathrm{rec}}}{t_{p r}}\right)^{2 / 3} \frac{1}{t_{c r}}
\end{aligned}
$$

(where $t_{\text {rec }}=1.2 \times 10^{13} \mathrm{sec}$ is the recombination time) the inflationary-GW power spectrum is distorted, departing from scale invariance.

We note that $f_{c}^{p r}$ depends only on the (physical) time at which the cosmic strings are formed. These linear defects may have been formed at a GUT transition or, conceivably, much later, at the electroweak transition or somewhere in between [4]. For GUT-scale strings, $t_{c r} \sim 10^{-31} \mathrm{sec}$ [5] and therefore $f_{c}^{p r} \simeq 1.5 \times 10^{5} \mathrm{~Hz}$. Clearly, this value is far outside of the range where both the ground-based and the space-based laser interferometers may operate. A GW of

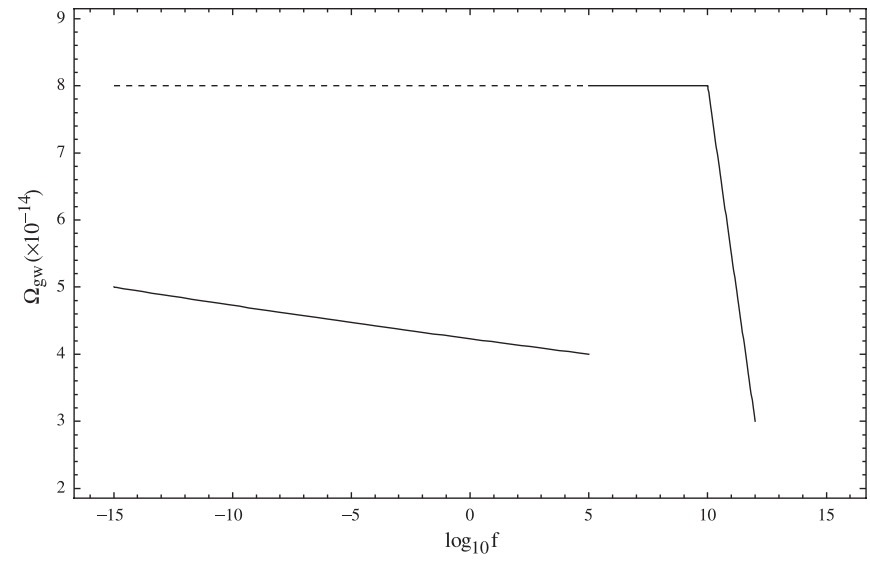

FIG. 1. The stochastic GW background from inflation at frequencies within the radiation era, $f \gtrsim 10^{-16} \mathrm{~Hz}$ (dashed line), under the influence of a radiation-plus-strings stage produced by GUT-scale cosmic strings (solid line). 
this frequency could be detected only by a system of coupled superconducting microwave cavities [40,41].

However, one should have in mind that, this is only the upper bound of the distorted GW power spectrum. In fact, if cosmic strings contribute to the evolution of the Universe, the GW power spectrum will decline from what it is anticipated by pure radiation at every presenttime frequency in the range $10^{-16} \mathrm{~Hz}<f \lesssim f_{c}^{p r}$ (see Fig. 1). The lower bound of this range arises from the GWs that began entering inside the horizon after the Universe has become matter dominated [39].

On the other hand, for electroweak-scale strings, $t_{c r} \sim$ $10^{-11} \mathrm{sec}[5]$ and hence, $f_{c}^{p r} \simeq 1.5 \times 10^{-5} \mathrm{~Hz}$, while, for cosmic strings created at some time in between the GUTand the electroweak-symmetry breaking (e.g., $t_{c r} \sim$ $10^{-21} \mathrm{sec}$ ), we obtain $f_{c}^{p r} \simeq 1.5 \mathrm{~Hz}$. Therefore, a potential detection of CGWs, among other things, would give us valuable information on the epoch (and therefore on the physical mechanism, as well) at which the cosmic strings were formed.

\section{CONCLUSIONS}

The equation which governs the temporal evolution of a CGW in a Friedmann universe can be treated as the Schrödinger equation for a particle moving in the presence of the effective potential $V_{\text {eff }}=R^{\prime \prime} / R$. In the present article we show that, if there is a period where the effective potential is constant, this would lead to a critical value $\left(k_{c}\right)$ in the comoving wave number of the metric fluctuations, discriminating them into oscillating $\left(k>k_{c}\right)$ and nonoscillating $\left(k<k_{c}\right)$ modes. As a consequence, when the nonoscillatory modes lie outside the horizon do not freeze out, something that should be reflected in the inflationary-GW power spectrum.

This property is met in a radiation model contaminated by a fraction of cosmic strings. Therefore, if the cosmo- logical evolution includes a radiation-plus-strings stage, some of the long-wavelength GW modes (although being outside the Hubble sphere) continue to evolve. However, this stage (if ever existed) does not last very long, since, the cosmological evolution gradually results in the scaling of the cosmic-string network and, after some time $(\Delta \tau)$, the Universe enters in the late-radiation era.

In a radiation-dominated universe the metric perturbations of $k<k_{c}$ can enter the horizon, which now expands faster than their physical wavelength. However, the evolution of the nonoscillatory GW modes during $\Delta \tau$ (while they were outside the horizon) has modified their amplitude and, therefore, oscillation of these modes within the Hubble sphere, results in the distortion of the scaleinvariant GW power spectrum at present-time frequencies in the range $10^{-16} \mathrm{~Hz}<f \lesssim 10^{5} \mathrm{~Hz}$.

\section{ACKNOWLEDGMENTS}

This project was supported by the Greek Ministry of Education, through the PYTHAGORAS Program and by the Spanish Research Projects MEC FPA-2004-04582 and FPA-2007-66665 and DURSI 2005SGR00082.

Note added in proof.-A few days after this article was accepted for publication, a recent paper [42] dealing with the scaling of a cosmic-string network in an updated numerical fashion came to our attention. According to it, the duration of a potential radiation-plus-strings stage in terms of the conformal time (the dynamical range-as it is referred to) is: $\tau_{s c}=17 \tau_{*}$ (corresponding to a factor of 300 in the physical time). Adoption of this result would lead to a more evident distortion of the inflationary GW spectrum, modifying Eq. (49) to $\Omega_{\mathrm{gw}}^{\mathrm{rps}} \simeq 0.2 \Omega_{\mathrm{gw}}^{\mathrm{rad}} \simeq 1.6 \times$ $10^{-14}$. The authors would like to thank Mairi Sakellariadou for pointing this out.
[1] S. Weinberg, Gravitation and Cosmology (Wiley, New York, 1972).

[2] M. Maggiore, Phys. Rep. 331, 283 (2000).

[3] L. P. Grishchuk and A. G. Polnarev, in General Relativity and Gravitation-One Hundred Years After the Birth of Albert Einstein, edited by A. Held (Plenum, New York, 1980).

[4] M. B. Hindmarsh and T. W. B. Kibble, Rep. Prog. Phys. 58, 477 (1995).

[5] A. Vilenkin and E.P.S. Shellard, Cosmic Strings and Other Topological Defects (Cambridge University Press, Cambridge, 2000).

[6] A. Vilenkin, Nature (London) 322, 613 (1986).

[7] Ya. B. Zel'dovich, Mon. Not. R. Astron. Soc. 192, 663
(1980).

[8] A. Vilenkin, Phys. Rev. Lett. 46, 1169 (1981).

[9] B. Allen, in The Stochastic Gravity-Wave Background: Sources and Detection, Les Houches School on Astrophysical Sources of Gravitational Waves, edited by J. A. Marck and J. P. Lassota (Cambridge University Press, Cambridge, 1997).

[10] C. W. Misner, K. S. Thorne, and J. A. Wheeler, Gravitation (Freeman, San Francisco, 1973).

[11] U. Bleyer, D. E. Liebscher, and A. G. Polnarev, Classical Quantum Gravity 8, 477 (1991).

[12] R. A. Battye, M. Bucher, and D. Spergel, arXiv:astro-ph/ 9908047.

[13] B. Carter, arXiv:hep-ph/0605029. 
[14] L. P. Grishchuk, Sov. Phys. JETP 40, 409 (1975).

[15] A. A. Starobinsky, JETP Lett. 30, 682 (1979).

[16] I. S. Gradshteyn and I. M. Ryzhik, Tables of Integrals, Series and Products (Academic Press, New York, 1965) [Eq. (8.491.5), p. 971].

[17] M. White, Phys. Rev. D 46, 4198 (1992).

[18] A. Vilenkin, Phys. Rev. D 24, 2082 (1981).

[19] A. Vilenkin, Phys. Rev. Lett. 53, 1016 (1984).

[20] T. B. W. Kibble, Phys. Rev. D 33, 328 (1986).

[21] T. B. W. Kibble, G. Lazarides, and Q. Shafi, Phys. Rev. D 26, 435 (1982).

[22] E. Witten, Phys. Lett. 153B, 243 (1985).

[23] S. Sarangi and S-H. H. Tye, Phys. Lett. B 536, 185 (2002).

[24] E.W. Kolb and M.S. Turner, The Early Universe (Addison-Wesley, New York, 1990).

[25] T. B. W. Kibble, J. Phys. A 9, 1387 (1976).

[26] T. B. W. Kibble, Phys. Rep. 67, 183 (1980).

[27] A. E. Everett, Phys. Rev. D 24, 858 (1981).

[28] A. Vilenkin, Phys. Rev. D 43, 1060 (1991).

[29] A. Albrecht and N. Turok, Phys. Rev. D 40, 973 (1989).

[30] D. P. Bennett and F. R. Bouchet, Phys. Rev. Lett. 63, 2776 (1989).
[31] D. P. Bennett and F. R. Bouchet, Phys. Rev. D 41, 2408 (1990).

[32] B. Allen and E. P. S. Shellard, Phys. Rev. Lett. 64, 119 (1990).

[33] L.F. Abbott and M. B. Wise, Nucl. Phys. B244, 541 (1984).

[34] J. E. Lidsey, A. R. Liddle, E. W. Kolb, E. J. Copeland, T. Barreiro, and M. Abney, Rev. Mod. Phys. 69, 373 (1997).

[35] L. P. Grishchuk, V. M. Lipunov, K. A. Postnov, M.E. Prokhorov, and B.S. Sathyaprakash, Phys. Usp. 44, 1 (2001).

[36] J. R. Pritchard and M. Kamionkowski, Ann. Phys. (N.Y.) 318, 2 (2005).

[37] V.F. Mukhanov, H. A. Feldman, and R. H. Brandenberger, Phys. Rep. 215, 203 (1992).

[38] B. J. Carr, Astron. Astrophys. 89, 6 (1980).

[39] W. Zhao and Y. Zhang, Phys. Rev. D 74, 043503 (2006).

[40] C. Reece, P. Reiner, and A. Melissinos, Phys. Lett. 104A, 341 (1984).

[41] C. Reece, P. Reiner, and A. Melissinos, Nucl. Instrum. Methods Phys. Res., Sect. A 245, 299 (1986).

[42] C. Ringeval, M. Sakelariadou, and F. R. Bouchet, J. Cosmol. Astropart. Phys. 02 (2007) 023. 